Revista Arbitrada Interdisciplinaria de Ciencias de la Salud. SALUD Y VIDA

Volumen 3. Número 1. Año 3. Edición Especial 2019

Hecho el depósito de Ley: FA2016000010

ISSN: $2610-8038$

FUNDACIÓN KOINONIA (F.K).

Santa Ana de Coro, Venezuela.

Ángel Luis Zamora Cevallos.

http://dx.doi.org/10.35381/s.v.v3i1.493

\title{
Seguridad alimentaria en la mala nutrición y discapacidad neurológica de niños entre 2 a 10 años
}

\section{Food safety in poor nutrition and neurological disability of children between 2 to 10 years}

\author{
Ángel Luis Zamora Cevallos \\ angelluisz2007@hotmail.com \\ Universidad Técnica de Manabí, Portoviejo \\ Ecuador. \\ https://orcid.org/0000-0001-8547-3592
}

Recibido: 12 de Julio de 2019

Aprobado: 15 de agosto de 2019

\begin{abstract}
RESUMEN
Entre la discapacidad neurológica y la nutrición, se puede dar una relación de dependencia de ambos sentidos. Bajo esta premisa se realizó este artículo cuyo objetivo fue evaluar la seguridad alimentaria en la mala nutrición y discapacidad neurológica de niños entre 2 a 10 años. La metodologia utilizada fue de tipo descriptiva - transversal. La población la constituyeron 44 niños con manifestaciones de mal nutrición y discapacidad neurológica, que asisten a las unidades operativas del distrito de salud 13D04 adscritos al Canton de Santa Ana en Manabí. El instrumento utilizado fue una guía de observación dicotómica. Entre sus resultados se destaca el hecho en la cual el grado de compromiso severo de la discapacidad neurológica se indicó en un $55 \%$ del total de los niños y el restante entre leve y moderado. Se concluye que existe una relación de correspondencia entre la alimentación con la discapacidad neurológica en los niños investigados.
\end{abstract}

Descriptores: Seguridad alimentaria; mala nutrición; discapacidad neurológica y salud.

\begin{abstract}
ABSTRAC
Between neurological disability and nutrition, a relationship of dependence of both senses can occur. Under this premise, this article was made, whose objective was to evaluate food safety in poor nutrition and neurological disability of children between 2 and 10 years. The methodology used was descriptive - transverse. The population consisted of 44 children with manifestations of poor nutrition and neurological disability, who attend the
\end{abstract}


Ángel Luis Zamora Cevallos.

operational units of the 13D04 health district attached to the Canton of Santa Ana in Manabí. The instrument used was a dichotomous observation guide. Among its results is the fact that the degree of severe commitment of neurological disability was indicated in $55 \%$ of the total children and the remainder between mild and moderate. It is concluded that there is a relationship of correspondence between feeding with neurological disability in the children investigated.

Descriptores: Food safety; poor nutrition; neurological disability and health.

\section{INTRODUCCIÓN}

Desde los años ' 80 , la desnutrición se abordó no sólo como un problema biológico, sino como la conjunción de factores económicos, antropológicos, sociológicos y culturales, convirtiéndose en un síndrome clínico, caracterizado por un insuficiente aporte de proteínas y/o calorías necesarias para satisfacer las necesidades fisiológicas del organismo tornándose incidente en las discapacidades neurológicas. A tal efecto, referirse a la seguridad alimentaria implica tener disposición a los alimentos, por parte de las personas y el aprovechamiento biológico de los mismos. Se considera que un hogar está en una situación de seguridad alimentaria cuando sus miembros disponen de manera sostenida a alimentos suficientes en cantidad y calidad según las necesidades biológicas. Sin embargo, estudios como el realizado por Piovani y col (2005), han comprobado que existe una vinculación entre el estado de desnutrición infantil y la afectación del crecimiento físico, bioquímico y mental, con numerosas consecuencias clínicas apreciadas desde el punto de vista morfológico y funcional.

Tambien, Aranceta (2013), explica que todo niño que ha sufrido desnutrición infantil evidencia un retraso en su crecimiento, en la talla y el peso corporal, en relación a la edad. Ha quedado ampliamente demostrado que las secuelas dejadas por la desnutrición se hacen evidentes en las alteraciones antropométricas, reducción del perímetro cefálico, talla baja, anemias, carencia de micro nutrientes, hipovitaminosis $A$, predisposición a contraer infecciones, alteraciones inmunológicas, trastornos a nivel del sistema nervioso 
central y disminución del Coeficiente Intelectual, patologías que han desencadenado en muchos casos en discapacidades neurológicas transitorias y/o permanentes.

Cabe destacar, que en algunos casos se da un exceso de malnutrición sobrevenida por la inactividad o el movimiento limitado que genera la discapacidad neurológica, sobre todo en aquellos niños que han adquirido una capacidad de alimentación completa, ocasionado desde los primeros años de vida, pudiendo no responder de la misma manera a la rehabilitación nutricional, como posiblemente lo haría el niño sano. Lo expuesto, permite describir que en el canton de Santa Ana, se indica casos de patologías clínicas de discapacidad neurológicas en niños asociadas a problemas de desnutrición alimentaria, los cuales en su mayoría no son atendidos en correspondencia a sus necesidades por la poca opción de sus familiares y/o padres de contar con los requerimientos de garantía de la seguridad alimentaria. Con base a esta posición, se planteó como objetivo del presente artículo evaluar la seguridad alimentaria en la mal nutrición y discapacidad neurológica de niños entre 2 a 10 años.

\section{DESARROLLO}

La relación entre discapacidad neurológica y nutrición, plantea Le Roy y col (2010), puede darse en ambos sentidos, en cuanto la enfermedad neurológica o su tratamiento pueden llevar a un compromiso nutricional global o de nutrientes específicos, o bien que las alteraciones nutricionales pueden afectar la evolución de la enfermedad neurológica y la respuesta al tratamiento. El abordaje de este binomio, según García, M (2005), puede ser considerado desde tres puntos de vista:

a.-La desnutrición como factor condicionante de deficiencias nutricionales que a su vez condicionan discapacidades neurológicas, motóricas o discapacidades.

b.- Exceso de nutrientes como fuente de patologías que tienen su base en una alimentación errónea por la transición nutricional que afecta a todas las personas, convirtiéndose en el origen de gran parte de las enfermedades crónicas y degenerativas que finalmente producirán discapacidades.

c.- Trastornos del comportamiento alimentario. 
Ángel Luis Zamora Cevallos.

d.- El cuarto aspecto es cómo alimentar a personas con discapacidad mediante la Alimentación Básica Adaptada y ayudas técnicas e instrumentales para la alimentación de personas con discapacidad.

Las enfermedades neurológicas pueden producir discapacidades motoras, sensitivas, sensoriales, musculares, cognitivas, conductuales o una mezcla de ellas, de diferente grado de compromiso o severidad. En la tabla 1, se presentan los principales problemas asociados a las enfermedades neurológicas y nutricionales.

Tabla 1. Problemas nutricionales asociados y bases de manejo de enfermedades neurológicas seleccionadas

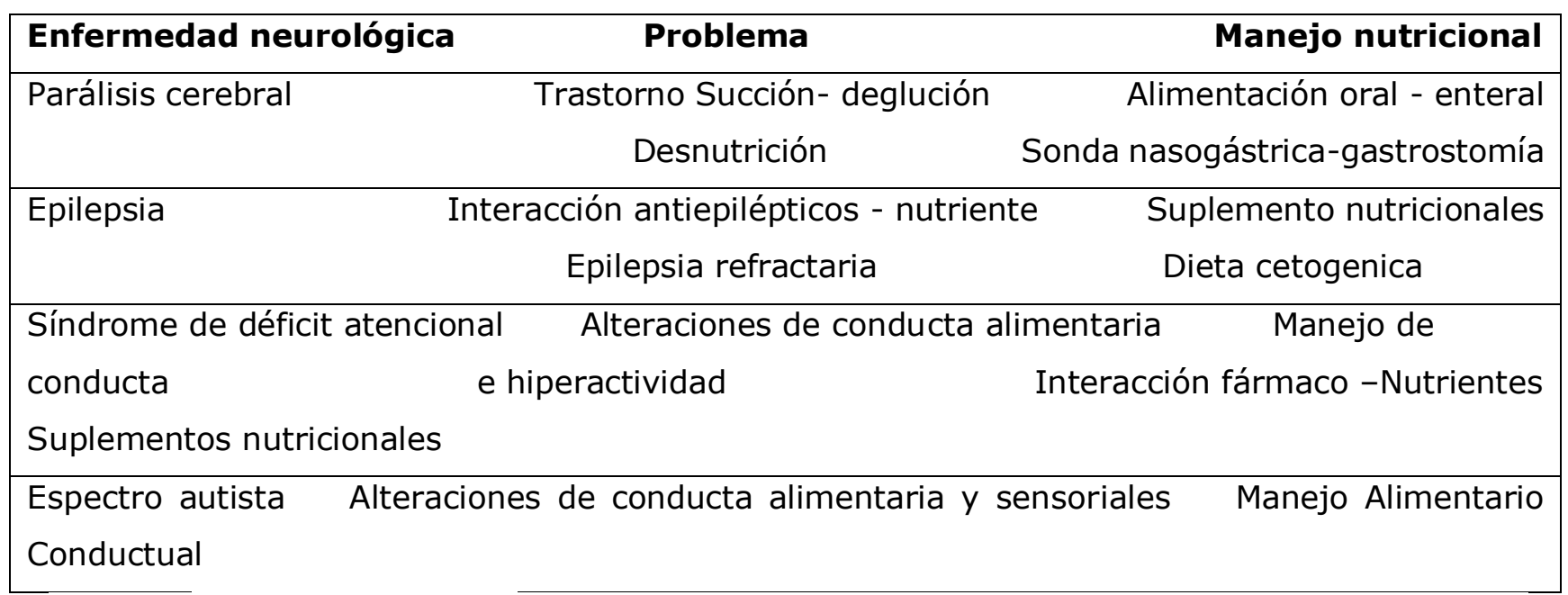

Fuente: Le Roy y col (2010)

Los mismos autores, exponen que los problemas nutricionales más frecuentes son: dificultad para consumir alimentos adecuados en cantidad y calidad, aspiración a vía aérea, desnutrición, sobrepeso u obesidad, deficiencia de macro y micronutrientes, osteoporosis. Además se presentan dificultades para realizar una evaluación nutricional adecuada por la limitación de los estándares de crecimiento disponibles para este grupo, el cual posee características de crecimiento y composición corporal específicas. 
En este mismo orden, Sullivan (2008) explica que los factores involucrados en estos problemas nutricionales son: alteraciones motoras (gruesa y fina), grado de ambulación y dependencia de sus cuidadores, alteraciones sensoriales, el tiempo de evolución y la patología de base. Las alteraciones gastrointestinales son frecuentes y muy importantes en esta enfermedad; dentro de ellas se encuentran la disfunción motora oral, con la dificultad secundaria para alimentarse, riesgo de aspiración por trastornos de deglución, tiempos de alimentación prolongados, reflujo gastroesofágico, retraso en el vaciamiento gastrointestinal, dis-motilidad intestinal y constipación.

\section{Consecuencias y características de una mala nutrición infantil.}

Las consecuencias de una mala alimentación son más serias de lo que se supone, especialmente el desarrollo de enfermedades graves. Para evitar esos efectos nocivos es necesario una buena educación y conocimientos en el campo de la nutrición. Las dietas sin contenido de vitaminas B,C,D y E y ácidos omega-3 atentan contra el buen funcionamiento cerebral, mientras que las altas en grasas trans, aceleran el envejecimiento cerebral, empeorando por tanto su correcto funcionamiento, (Aranceta, 2013). A este hecho, se suman la malnutrición, que no es lo mismo que desnutrición, presentando alteraciones en la piel, trastornos en la pigmentación del cabello, problemas de visión, enferman fácilmente y no se desarrollan adecuadamente ni física ni mentalmente, y en el peor de los casos discapacidades hasta de tipo neurológicas.

Entre sus características, se destacan según Barboza (2000), una de leve, medio y severa desnutrición.

Desnutrición leve: es la que más padece la población infantil, pero también en lo general, a la que menos atención se le brinda; por ello es conveniente mencionar que entre más tempranamente ocurra y se prolongue por mayor tiempo, más daños ocasionará, ya que esto no sólo se restringe a la disminución del tamaño corporal, sino también afecta el desarrollo y propicia la frecuencia y gravedad de enfermedades de tipo neurologicas. La alimentación que reciba el niño con desnutrición leve, debe tener las 
Ángel Luis Zamora Cevallos.

características de una dieta normal, pero fraccionadas en mayor número de comidas al día. Para que tanto el tratamiento dietético como la rehabilitación sean exitosos y el niño se recupere en corto tiempo, es fundamental que el personal de salud y las personas que lo atienden tengan conocimientos de que los alimentos y una atención especial son los que hacen posible la recuperación.

Desnutrición Moderada: Es cuando ha avanzado el déficit de peso y generalmente tiene patologías agregadas, principalmente enfermedades de las vías intestinales y respiratorias. Su magnitud le sigue en importancia a la desnutrición leve. Cuando el niño cursa este tipo de desnutrición los signos y síntomas son más acentuados, mencionándose los siguientes:

El niño se muestra apático y desganado por lo cual se cansa hasta para comer, no siente hambre y no demanda su alimentación. Por lo que los padres o personal de salud deben insistir en proporcionarle los alimentos poco voluminosas, nutritivas, 6 a 7 veces al día, en intervalos de $21 / 2$ a 3 horas.

Desnutrición Severa: En sus diversas manifestaciones, es sin duda la más dramática de las enfermedades nutricionales; generalmente se identifica en dos tipos, Kwashiorkor y Marasmo. Las metas del tratamiento deben tener como finalidad en primera instancia, recuperar las deficiencias específicas, tratar las infecciones, suprimir la flora gastrointestinal anormal y revertir las deficiencias funcionales adaptativas. Paralelamente proporcionar una dieta que permita la rápida recuperación de los tejidos perdidos y el restablecimiento de la composición corporal normal. La dieta debe ser proporcionada en forma gradual por etapas según la evolución del niño. Inicialmente se debe efectuar de manera inmediata y adecuada, las medidas tendientes a tratar las complicaciones de la desnutrición severa. De esta forma debe atenderse el desequilibrio electrolítico y cualquier otro padecimiento que se tenga (gastroenteritis, bronconeumonía, u otro). 


\section{Discapacidad neurológica}

La discapacidad neurológica, explica García y col (2011), se asocia a trastornos o alteraciones que se desarrollan en la zona cerebral. Pueden afectar tanto al sistema nervioso central como al sistema neurológico. Esta discapacidad puede alterar actividades para las que se precisa la funcionalidad correcta del cerebro como la memoria, el habla, las actividades motoras y sensoriales, entre otras.

Vásquez, (2008), indica que la discapacidad neurológica es una enfermedad congénita o adquirida a largo plazo asociada con limitaciones funcionales que se atribuyen a un trastorno del cerebro y/o del sistema neuromuscular. Estas incluyen: Parálisis cerebral. Trastorno del espectro autista. Epilepsia. Distrofia muscular de Duchenne. Tales situaciones clínicas pueden conducir a convulsiones e hipotonía muscular (fuerza muscular reducida), que pueden afectar a la capacidad del niño para comer. De hecho, hasta un $40 \%$ de los niños con discapacidades neurológicas sufren disfunción oral motora, lo cual puede conducir a dificultades en la alimentación, tales como el cierre incompleto de los labios, babeo excesivo (y pérdida de líquidos), reducida lateralización de la lengua, deglución descoordinada, asfixia y tos.

Asimismo, en pacientes con discapacidad neurológica existen dificultades para garantizar una adecuada nutrición, generando en ellos inadecuada evaluación nutricional, alta frecuencia de bajo peso, fallo de crecimiento, deficiencia de micronutrientes, osteopenia, dificultades en la suplementación oral, aspectos parentales, y entre las alteraciones crónicas del sistema central nervioso trastornos motores orales o disfunción de la deglución, parálisis cerebral y defectos genéticos, entre otros. (García, 2005)

Moreno y col (2001), indica que un adecuado estado nutricional en pacientes con discapacidad neurológica, se asocia con mejor salud mental y calidad de vida, se dan menor frecuencia de hospitalizaciones, de inasistencias a rehabilitación o escolares, progresos en el desarrollo y mejor perfusión periférica. Tambien, existe en estos niños con discapacidad neurológica una deglución alterada, succión débil, falta de lateralización de la lengua, extrusión persistente de la lengua, mala oclusión de los labios, incapacidad 
de formar el bolo alimenticio, prensión defectuosa de los alimentos, todos estos aspectos mencionados son causantes de la malnutrición en pacientes con esta patología.

Por otra parte, se requieren padres y/o cuidadores que comprendan las señales de sus hijos por los problemas en la comunicación del niño para poder expresar que tienen hambre, sed y/o preferencias de comidas, asi como el poseer una actividad física disminuida, mala posición en la silla, inadecuado sostén del tronco, silla que no lo mantiene en una postura correcta, inmadurez neurológica son motivo de desnutrición que no les favorece para la mejora de su incapacidad neurológica. (García, 2005)

\section{METODOLOGÍA}

La metodologia utilizada fue de tipo descriptiva. Según Hernández y col (2014), este tipo de método descriptivo de investigación es el procedimiento usado en ciencia para describir las características del fenómeno, sujeto o población a estudiar. Asimismo, se estableció una metodología documental, la cual se basó en la obtención y análisis de datos bibliográficos y no bibliográficos u otros tipos de documentos teórico relacionado con la materia en estudio (Parreño, 2016). Así mismo Chávez (2011), expone que el diseño de la investigación consiste en una estrategia o plan general que permite las operaciones necesarias para lograr el estudio del fenómeno. Para la obtención de los datos e informacion se utilizó una guía de observación dicotómica.

Asimismo, se consideró según su temporalidad transversal, en tanto la informacion y/o datos se tomaron en un solo momento. La población la constituyeron 44 niños con manifestaciones de mal nutrición y discapacidad neurológica, que asisten a las unidades operativas del distrito de salud 13D04 adscritos al canton de Santa Ana en Manabí. Los criterios de inclusión se correspondieron a niños entre 2 a 10 años que asisten a las unidades operativas acompañados de sus padres y/o representantes para asistencia nutricionales asociadas con discapacidad neurológica. Asimismo, se solicitó el apoyo de un fisioterapeuta que requerían aquellos niños para las maniobras a realizar según las particulares del niño/a. 
Revista Arbitrada Interdisciplinaria de Ciencias de la Salud. SALUD Y VIDA

Volumen 3. Número 1. Año 3. Edición Especial 2019

Hecho el depósito de Ley: FA2016000010

ISSN: $2610-8038$

FUNDACIÓN KOINONIA (F.K).

Santa Ana de Coro, Venezuela.

Ángel Luis Zamora Cevallos.

\section{RESULTADOS}

Posterior al análisis de la informacion obtenida por parte de los informantes de los 44 niños investigados en las unidades operativas del distrito de salud 13D04 adscritos al Canton de Santa Ana en Manabí, entre lo que se pudo constatar:

-Se identificaron 44 menores con edades entre 2 a 10 años, con un promedio de edad entre 3.5 y 8.2 años, con un ligero predominio del sexo masculino (56\%) sobre el femenino (44\%). El diagnóstico clínico predominante en ambas sexos malnutrición y discapacidad neurológica. En cuanto al grado de compromiso con la edad, casi el 77\% de los casos presentaban una desnutrición y discapacidad entre grave (32\%) y moderada (43\%), correspondiendo solo un $25 \%$ para de los casos leves.

-Con relación al grado de compromiso de la discapacidad neurológica, se observó un predominio de niños con un compromiso severo (55\%), el $45 \%$ restante fueron niños afectados con un compromiso entre leve y moderado.

-Para el desarrollo motor, se indica un predominio de niños con un desarrollo motor que les permitía en posición sentado mantener la cabeza derecha (72.5\%), solo 14 niños no la mantenía derecha y 2 niños se encontraron en posición acostado.

-La utilización de los miembros superiores, en el $41 \%$ de los niños los tienen inútiles, correspondiéndose a un 59\% los niños calificados para las categorías poco útiles y útiles. En cuanto a la utilización de los miembros inferiores, existe un alto porcentaje de niños con el $54 \%$ que no caminan y el $38 \%$ caminaban por si solos y el $8 \%$ ayudados por bastones ortopédicos o silla de ruedas para desplazarse.

En cuanto a la capacidad de alimentación, los menores presentaban una capacidad de alimentación completa (82\%), indicando buenas condiciones para ingerir los alimentos de acuerdo a todas las texturas: líquida, semilíquida, semisólida y sólida. Solo el $18 \%$ presentó una capacidad de alimentación incompleta. De ellos, un 11\% toleraba alimentos con una consistencia líquida y semilíquida.

-Los niños con problemas de nutrición se indicaron con mayor frecuencia $(55 \%)$ entre las edades de 2 a 4 años, respectivamente. En esta submuestra estudiada, se pudo 
Ángel Luis Zamora Cevallos.

evidenciar que su estado nutricional incidía sobre su tono muscular, el grado de compromiso, el desarrollo motor en miembros superiores y la capacidad de alimentación. -Se pudo constatar, que en el conjunto de niños con déficit nutricional y riesgo nutricional, el $75 \%$ tenía un tono muscular espástico y un grado de compromiso severo. De ellos, el $60 \%$ presentó más de un trastorno asociado y a su vez, en la mayoría se relacionaba con problemas de la capacidad de alimentación.

\section{CONCLUSIONES}

Se puede indicar una relación de correspondencia entre la alimentación con la discapacidad neurológica en los niños investigados, explícitamente en el grado o nivel de discapacidad motora y la tolerancia de alimentación asociado a su tono muscular y desarrollo motor en miembros superiores e inferiores.

Este estudio confirma que los niños con mal nutrición y discapacidad neurológica requieren de una atención especializada y personalizada en forma permanente para contribuir a minimizar los trastornos de la capacidad de alimentación y mejorar su calidad de vida.

Es necesario conjugar las particularidades de cada cuadro clínico y la capacidad para alimentarse con la finalidad de garantizar una alimentación acorde a las necesidades de cada uno de los niños evaluados evitando agravar su estado nutricional.

En cuanto a los niños con serias dificultades para mantenerse en equilibrio su canal alimentario, se puede observar un marcado grado de desnutrición, debido a la poca efectividad de su proceso de deglución, demandando más atención durante su proceso de alimentación por parte de sus padres, con la finalidad de dar garantía de la cantidad y calidad requerida según el caso tratado. 
Ángel Luis Zamora Cevallos.

\section{REFERENCIAS CONSULTADAS}

1. Aranceta, J. (2013). Nutrición comunitaria. $3^{\circ}$ ed. Buenos Aires: Elsevier España. p. 136-149.

2. Barboza, C (2000). Es fatal el retardo mental? Discapacidad severa y grave. $2^{\underline{a}}$ parte. Revista Futuro. Panamá. jun.No.5.

3. Chávez, N. (2011) Introducción a la Investigación Educativa. Maracaibo, Estado Zulia. Editora La Columna. 5ta Edición.

4. García, T (2005). Nutrición y discapacidad. Importancia y posibilidades de prevención. Escuela Nacional de Sanidad. Instituto de salud Carlos III. España

5. García, L y Restrepo, S. (2011). Alimentar y nutrir a un niño con parálisis cerebral. Una mirada desde las percepciones. Invest Educ. Enferm. 29(1):28-39.

6. Hernández R, Fernández C, Baptista L. (2014). Metodología de la Investigación. México: Editorial Mc Graw Hill.

7. Le Roy, C; Rebollo, M; Moraga, F; Diaz Ximena y Castillo, C (2010). Nutrición del Niño con Enfermedades Neurológicas Prevalentes. Versión impresa ISSN 03704106. http://dx.doi.org/10.4067/S0370-41062010000200002.

8. Moreno, JM; Galiano MJ; Valero Z; León Sanz, M.(2001). Alimentación en el paciente con parálisis cerebral. Nutrición infantil. Acta Pediátrica Española. 59(1):17-25.

9. Parreño, Á. (2016). Metodología de la Investigación en Salud. Riobamba: Escuela Superior Politécnica de Chimborazo.

10. Piovani, V y Piovani, M. (2005). Anemia en niños y adolescentes. Revista de la Fundación Argentina contra la Anemia. Año 1, número 1, abril/05.

11. Sullivan, P (2008). Gastointestinal disorders in children with neurodevelopmental disabilities. Dev Disabil Res Rev 2008; 14: 128-36.

12. Vásquez, A. (2008). Situación de la discapacidad en las Américas. In: Organización Panamericana de la Salud. El abordaje de la discapacidad desde la atención primaria de la salud. Buenos Aires: OPS; p. 20-25. 
Ángel Luis Zamora Cevallos.

\section{REFERENCES CONSULTED}

1. Aranceta, J. (2013). Community nutrition 3rd ed. Buenos Aires: Elsevier Spain. p. 136-149.

2. Barboza, C (2000). Is mental retardation fatal? Severe and severe disability. 2nd part. Future Magazine. Panama. Jun.No.5

3. Chavez, N. (2011) Introduction to Educational Research. Maracaibo state zulia. The Column Editor. 5th Edition.

4. García, T (2005). Nutrition and disability Importance and prevention possibilities. National School of Health. Carlos III Health Institute. Spain

5. García, L y Restrepo, S. (2011). Feed and nourish a child with cerebral palsy. A look from perceptions. Invest Educ. Sick. 29 (1): 28-39.

6. Hernández R, Fernández C, Baptista L. (2014). Investigation methodology. Mexico: Editorial Mc Graw Hill.

7. Le Roy, C; Rebollo, M; Moraga, F; Diaz Ximena y Castillo, C (2010). Nutrition of the Child with Prevalent Neurological Diseases. Printed version ISSN 0370-4106. http://dx.doi.org/10.4067/S0370-41062010000200002.

8. Moreno, JM; Galiano MJ; Valero Z; León Sanz, M. (2001). Feeding in the patient with cerebral palsy. Child nutrition. Spanish Pediatric Act. 59 (1): 17-25.

9. Parreño, Á. (2016). Methodology of Health Research. Riobamba: Polytechnic School of Chimborazo.

10. Piovani, V and Piovani, M. (2005). Anemia in children and adolescents. Magazine of the Argentina Foundation against Anemia. Year 1, number 1, April / 05.

11. Sullivan, P (2008). Gastointestinal disorders in children with neurodevelopmental disabilities. Dev Disabil Res Rev 2008; 14: 128-36.

12. Vásquez, A. (2008). Situation of disability in the Americas. In: Pan American Health Organization. The approach to disability from primary health care. Buenos Aires: PAHO; p. 20-25.

(C2019 por los autores. Este artículo es de acceso abierto y distribuido según los términos y condiciones de la licencia Creative Commons Atribución-NoComercial-Compartirlgual 4.0 Internacional (CC BY-NC-

SA 4.0) (https://creativecommons.org/licenses/by-nc-sa/4.0/). 